\title{
Effects of excitonic diffusion on stimulated emission in nanocrystalline $\mathrm{ZnO}$
}

\author{
Ger Tobin, Enda McGlynn, a) Martin O. Henry, and Jean-Paul Mosnier \\ School of Physical Sciences/National Centre for Plasma Science and Technology, \\ Dublin City University, Glasnevin, Dublin 9, Ireland \\ Eduardo de Posada and James G. Lunney \\ School of Physics, Trinity College Dublin, Dublin 2, Ireland
}

(Received 20 July 2005; accepted 3 January 2006; published online 17 February 2006)

We present optically pumped emission data for $\mathrm{ZnO}$, showing that high excitation effects and stimulated emission/lasing are observed in nanocrystalline $\mathrm{ZnO}$ thin films at room temperature, although such effects are not seen in bulk material of better optical quality. A simple model of exciton density profiles is developed which explains our results and those of other authors. Inhibition of exciton diffusion in nanocrystalline samples compared to bulk significantly increases exciton densities in the former, leading - via the nonlinear dependence of emission in the exciton bands on the pump intensity - to large increases in emission and to stimulated emission. () 2006 American Institute of Physics. [DOI: 10.1063/1.2174107]

$\mathrm{ZnO}$ has recently emerged as a strong candidate for ultraviolet photonic devices, with attractive properties, including a large exciton binding energy of $60 \mathrm{meV}$ enabling efficient excitonic emission at room temperature. Many groups have studied stimulated emission and lasing properties of bulk $\mathrm{ZnO}$, under electron beam ${ }^{1}$ and optical pumping, ${ }^{2}$ but evidence for stimulated emission and lasing has only been seen at rather low temperatures. Recent studies of optically pumped nanocrystalline samples reveal stimulated emission and lasing even at room temperature, in contrast to the bulk studies, even in samples of similar or inferior quality compared to bulk crystals. ${ }^{3-6}$

We have studied optically pumped emission at room temperature from bulk single-crystal material (Eagle-Picher Corporation) and samples grown by pulsed laser deposition (PLD) on $c$ sapphire. Details of the sample growth are given elsewhere. ${ }^{7}$ Some films were annealed in $\mathrm{O}_{2}(0.3 \mathrm{mbar})$ between $400{ }^{\circ} \mathrm{C}$ and $600{ }^{\circ} \mathrm{C}$ in the growth chamber after deposition leading to a consistent increase in grain size (30 $\rightarrow 80 \mathrm{~nm}$ ) with annealing temperature. ${ }^{7}$ All films show evidence of electric-field damping of the free exciton (FE) and film thicknesses are in the range of $200-250 \mathrm{~nm} .^{7,8}$ The samples were studied using continuous-wave photoluminescence (PL) and high excitation pumping with a frequencytripled Nd:YAG laser $(355 \mathrm{~nm} 10 \mathrm{~Hz} 6 \mathrm{~ns}$ pulse width) focused on the sample to a diameter of $\sim 1.2 \mathrm{~mm}$ (determined by the knife-edge method) enabling excitation intensities in the range of $500-7000 \mathrm{~kW} / \mathrm{cm}^{2}$. Full details are described in Refs. 7 and 9.

Figure 1(a) shows emission at $300 \mathrm{~K}$ from a PLD sample (annealed at $500{ }^{\circ} \mathrm{C}$ ) as a function of increasing $\mathrm{Nd}$ :YAG excitation power. The data from this sample are representative of all the PLD samples. With increasing excitation, one observes the growth first of the FE band at $3.33 \mathrm{eV}$ and then the $\mathrm{P}$ band at $3.23 \mathrm{eV}$, corresponding to the well-known exciton-exciton collision process, $\sim 100 \mathrm{meV}$ below the FE. ${ }^{2}$ The emission bands are significantly broadened due to the damping effects mentioned earlier. The plot

\footnotetext{
a) Author to whom correspondence should be addressed; electronic mail:
} enda.mcglynn@dcu.ie of integrated emission intensity versus excitation power shown in Fig. 1(b) exhibits a distinct threshold at a pump power slightly above $2000 \mathrm{~kW} / \mathrm{cm}^{2}$, which is not explained by a superlinear power-law dependence of emission on pump level. Lasing cavity modes are seen at pump powers above this value, while no such modes are seen at lower pump

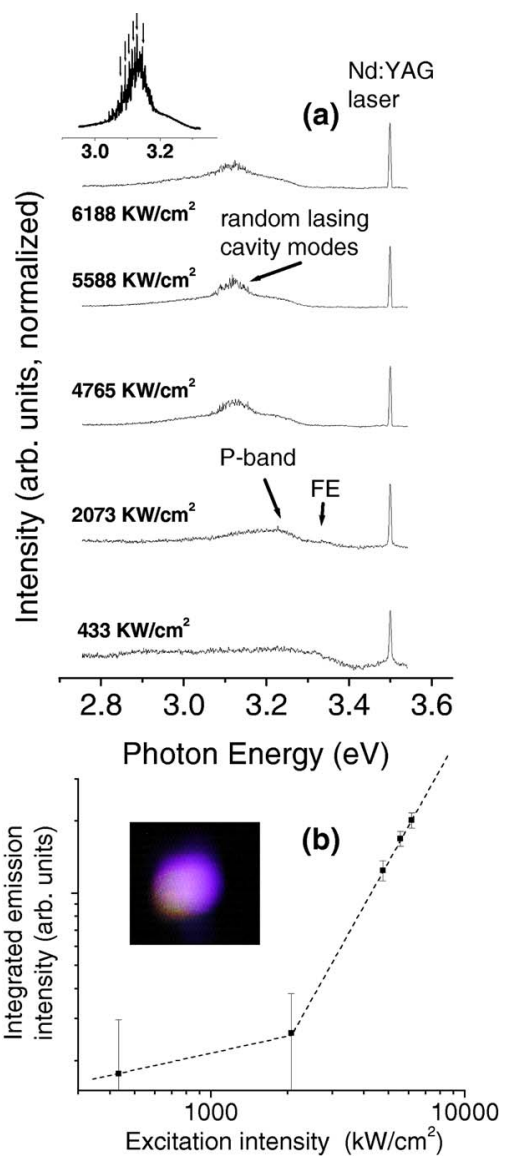

FIG. 1. (a) Emission spectra vs excitation power for PLD sample at room temperature (all spectra normalized to maximum intensity). Inset shows mode structure above threshold. (b) Integrated emission intensity vs excitation power (both axes displayed logarithmically). Dashed line is a guide for the eyes. The intensity integration is over the range $2.82 \mathrm{eV}$ to $3.45 \mathrm{eV}$. The speckled appearance of the sample surface is shown in the inset. 

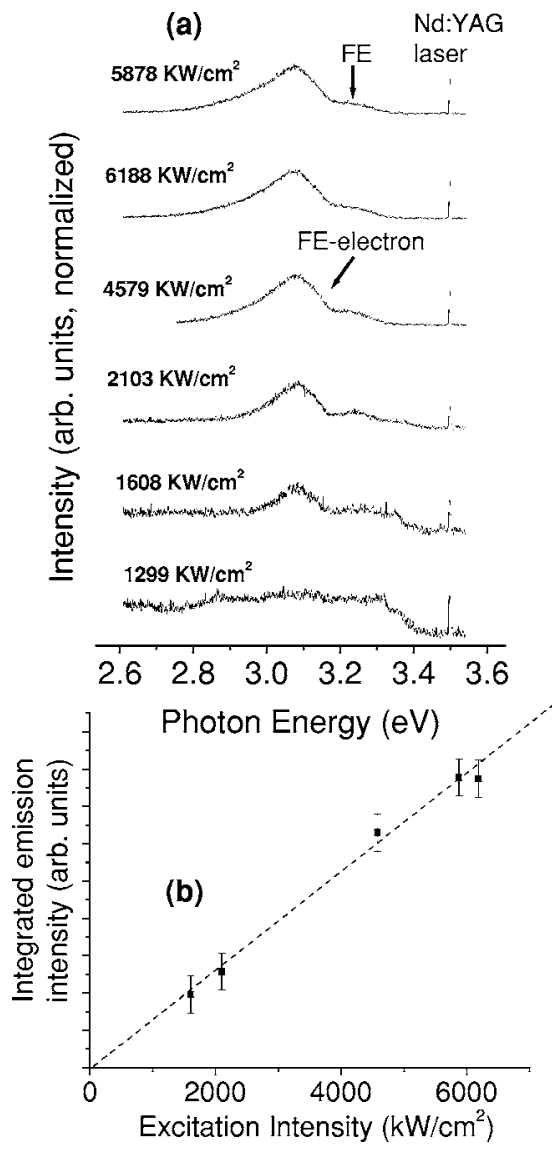

FIG. 2. (a) Emission spectra vs excitation power for bulk crystal at room temperature (all spectra normalized to maximum intensity). (b) Integrated emission intensity vs excitation power. The intensity integration is over the range $2.82 \mathrm{eV}$ to $3.45 \mathrm{eV}$. Dashed line is a guide for the eyes.

powers. Simultaneously, we note the appearance of a visible "speckled cloud" of stimulated emission [inset of Fig. 1(b)] on the sample surface, demonstrating the occurrence of random lasing. ${ }^{10} \mathrm{ZnO}$ bulk material exhibits no evidence of stimulated emission at $300 \mathrm{~K}$ (Fig. 2). At low excitation densities, we observe FE and deep level luminescence ${ }^{11}$ while at higher excitation, we observe the growth of a broad band due to the exciton-electron interaction ( $n$-type concentration in these samples $\left.\sim 10^{17} \mathrm{~cm}^{-3}\right){ }^{2}$ A plot of integrated emission intensity versus excitation reveals linear behavior with no threshold and no evidence of coherent emission or a specklelike appearance was seen.

Other authors have noted that poly- or nanocrystalline thin films, unlike bulk $\mathrm{ZnO}$ material, exhibit low thresholds for the appearance of nonlinear emission bands and optically pumped stimulated emission. ${ }^{3-6}$ The origin of this behavior compared to bulk material has been attributed to giant excitonic oscillator strengths in low-dimensional nanocrystalline structures, based on theoretical studies. ${ }^{3,12}$ Reflectance data on our samples, however, show a reduction in the oscillator strengths (i.e., a reduction in the longitudinal-transverse splitting, $\Delta_{\mathrm{LT}}$ ) compared to bulk material, in addition to increased exciton damping. ${ }^{8,13}$ Additionally, the nanocrystalline films show mediocre PL quality compared to bulk material. Other reported linear optical measurements (e.g., PL, absorption, and reflectance) on poly- or nanocrystalline $\mathrm{ZnO}$ which show lasing at room temperature also reveal no evidence of giant oscillator strengths, ${ }^{3-6,14}$ similar to our data. Various authors have suggested that the wave-guiding prop-
Downloaded 09 Jul 2009 to 134.226.1.229. Redistribution subjec erties of the air/ZnO/sapphire structure affect the stimulated emission properties leading to large gains in thin films. ${ }^{15}$ The critical film thicknesses identified are $\sim 50-60 \mathrm{~nm}$, substantially thinner than our films. Hence, we do not believe that this effect is appropriate to explain our data either.

A simpler explanation, not suggested before in this context, is that the FE density in nanocrystalline materials may be substantially different than that in bulk material due to the inhibition of FE diffusion in nanocrystalline samples at grain boundaries, similar to effects seen in thin films. ${ }^{16,17}$ The Pand electron-hole pair bands-in which stimulated emission and lasing are normally seen-grow in proportion to the second (or higher) powers of the FE density ${ }^{2}$ and are thus very sensitive to the density. In nanocrystalline samples, the FE population created by optical absorption in the $\sim 50 \mathrm{~nm}$ penetration depth ${ }^{18}$ will be strongly confined in this volume by grain boundaries. ${ }^{4}$ In contrast, FE diffusion lengths in bulk material may be significantly larger. FE diffusion lengths at low temperature $(<77 \mathrm{~K})$ are reported in the range of $0.2-2 \mu \mathrm{m},{ }^{16,19,20}$ and hence the initial exciton population will diffuse and lower its density substantially. This explanation assumes that FE are confined in the grain volume by grain boundaries but that nonradiative recombination due to deep levels at the grain boundary do not strongly quench the FE. This implies that grain boundaries repel FEs, and that FEs do not approach the boundary close enough for a high degree of nonradiative recombination. There is strong evidence throughout the literature supporting this idea, showing that high intensity PL emission (and lasing) is seen from nanocrystalline $\mathrm{ZnO}$ thin films even when the grain structure is pronounced. ${ }^{4}$ The mechanism of repulsion will probably be related to a variety of physical causes, but space-charge layers close to grain boundaries, associated with charge trapping at the boundaries, may be particularly important. Electric fields in these space-charge regions are known to be important in understanding the properties of $\mathrm{ZnO}$ thin films, and these samples in particular. ${ }^{7,9}$ Electric fields of a magnitude expected at grain boundaries increase the FE energy, i.e., the exciton is repelled. ${ }^{7,21}$ There is an associated quenching of the FE due to the field, mentioned above, but the dominant effect of the field is to repel the FE from grain boundaries toward low-field regions. The small FE Bohr radius $(2 \mathrm{~nm})$ in $\mathrm{ZnO}$ (Ref. 11) means that the electron and hole do not interact directly with the deep levels at the boundary when the FE center of mass is confined to the field-free region, as the space-charge regions are larger than the FE Bohr radius. ${ }^{7}$ This mechanism explains why the excitons are confined to the grain volume, without being strongly quenched by deep levels at the boundaries.

We use a 1D model to explore this effect for the PLD thin film and bulk samples (since the laser spot size diameter is much larger than the penetration depth of the FE population). The FE generation rate per unit area $(G)$ due to the pump laser (assuming unit quantum efficiency) incident on the PLD samples is equated to the FE recombination rate per unit area $(R)$ for steady state. In these samples, excitons are assumed to be confined with uniform density in the penetration depth $(\sim 50 \mathrm{~nm}$, which is also typical of the sample grain sizes), represented by $T$ in Fig. 3. The equilibrium concentration of excitons created in the polycrystalline material is given by

to AIP license or copyright; see http://apl.aip.org/apl/copyright.jsp 


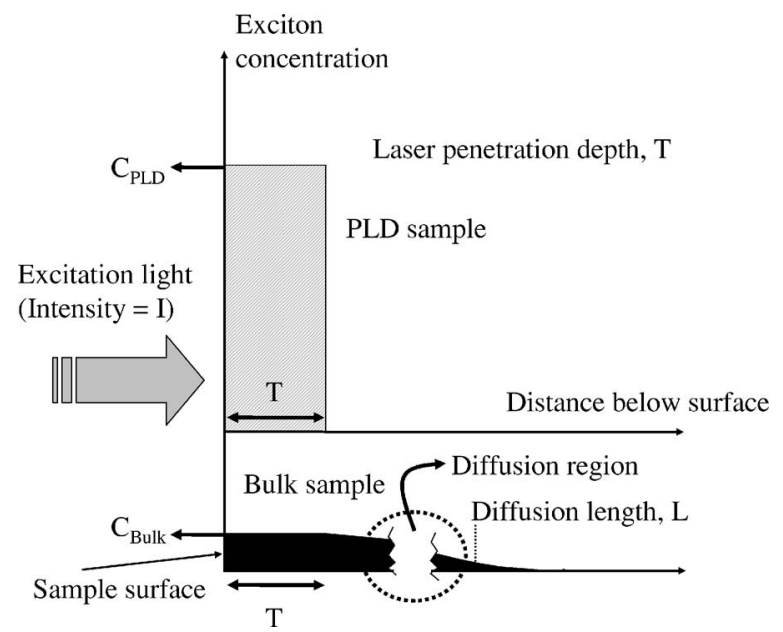

FIG. 3. Schematic diagram of concentration profile of excitons in bulk and PLD samples (vertical scale appropriate for $L=7 T$ ).

$$
G=\frac{I}{h f}=R=\frac{C_{\mathrm{PLD}} T}{\tau} \Rightarrow C_{\mathrm{PLD}}=\frac{\tau}{T}\left(\frac{I}{h f}\right),
$$

where $I$ is the intensity of the laser per unit area, $h$ is Planck's constant, $f$ is the frequency, $C_{\mathrm{PLD}}$ is the FE concentration at $x=0$, and $\tau$ is the FE lifetime. For bulk material, the generation rate of FE is the same as for PLD material in the penetration depth. We assume that in this depth the concentration is a constant, $C_{\mathrm{BULK}}$, and that beyond this region it decays with an exponential diffusion profile as in Fig. 3, where $L$ is the diffusion length of FE in bulk $\mathrm{ZnO}$. The rate of loss of FE per unit area from the penetration depth region may be expressed as the sum of the recombination and diffusion currents and equals $\left[C_{\mathrm{BULK}} T / \tau\right]+J_{D}$. The first term is similar to the PLD case and the second is the diffusion current density, $J_{D}$, from Fick's law. Equating this sum to the generation rate in the penetration depth gives the equilibrium maximum concentration of FE in the bulk, $C_{\mathrm{BULK}}$. The ratio of the maximum FE population levels for the bulk and the PLD material (using the relationship $L=\sqrt{D \tau}$ ) is:

$$
\begin{aligned}
C_{\mathrm{BULK}}= & I\left(h f\left(\frac{T}{\tau}+\frac{D}{L}\right)\right]^{-1} \\
& \Rightarrow \frac{C_{\mathrm{BULK}}}{C_{\mathrm{PLD}}}=\frac{T / \tau}{T / \tau+D / L}=\left(1+\frac{L}{T}\right)^{-1} .
\end{aligned}
$$

Taking the diffusion length $L$ at low temperatures to be $\sim 1 \mu \mathrm{m}$, the ratio of the concentrations in bulk and PLD material will be $\sim 0.05$. Thus, the superlinear optical emission bands may be at least two orders of magnitude stronger in the nanocrystalline PLD material based purely on diffusion effects. The FE diffusion length used is based on reports at low temperature. Given that such diffusion lengths increase with temperature in many systems, ${ }^{22}$ it may be regarded as a reasonable lower bound, implying that the ratio of bulk to PLD FE concentrations at 300K will not be greater than indicated in our calculation. Thus we expect that, based solely on diffusion effects, nonlinear emission bands and stimulated emission/lasing will be very weak in bulk material at room temperature compared to nanocrystalline material.

Low lasing thresholds $\left(\sim 40 \mathrm{~kW} / \mathrm{cm}^{2}\right)$ are seen at room temperature in some single-crystal $\mathrm{ZnO}$ nanowires, with lengths of $\sim 10 \mu \mathrm{m} .{ }^{23}$ Such nanowires would be expected to behave similarly to bulk material. However, these low thresholds have been reported only for nanorods grown by a metal-catalyzed (gold/self-catalyzed) process, leaving a metallic region at the nanowire tip. ${ }^{23}$ These effects can be explained by analogy with repulsive image charge effects known to efficiently exclude FE from a region close to the sample surface ("dead-layer"). ${ }^{16}$ Attractive image charge effects at a semiconductor-metal boundary may lead to FE localization close to the tip and locally high FE densities. Near-field scanning optical microscopy of single gold-tipped nanowires under high excitation ${ }^{23}$ shows light emission strongly localized at one end of the nanowire, suggesting such an exciton confinement.

In conclusion, we show evidence for high excitation effects and lasing in nanocrystalline $\mathrm{ZnO}$ thin films at room temperature, although such effects are not seen for bulk material of superior optical quality. Conventional explanations of such differences are not suitable to explain our results. We propose that the inhibition of FE diffusion in nanocrystalline thin films substantially increases FE densities, leading to stimulated emission and lasing. Calculations indicate differences greater than two orders of magnitude in $\mathrm{P}$ band and EHP emission and show why room-temperature lasing is seen only in nanocrystalline thin films, or other systems with local exciton confinement, rather than bulk material.

The authors acknowledge the support of an Enterprise Ireland Basic Research Grant and funding under NDP.

${ }^{1}$ J. M. Hvam, Phys. Rev. B 4, 4459 (1973).

${ }^{2}$ C. Klingshirn, Phys. Status Solidi B 71, 547 (1975).

${ }^{3}$ P. Zu, Z. K. Tang, G. K. L. Wong, M. Kawasaki, A. Ohtomo, H. Koinuma, and Y. Segawa, Solid State Commun. 103, 459 (1997).

${ }^{4}$ M. Kawasaki, A. Ohtomo, I. Ohkubo, H. Koinuma, Z. K. Tang, P. Yu, G. K. L. Wong, B. P. Zhang, and Y. Segawa, Mater. Sci. Eng., B 56, 239 (1998).

${ }^{5}$ X. Q. Zhang, I. Suemune, H. Kumano, J. Wang, and S. H. Huang, J. Appl. Phys. 96, 3733 (2004).

${ }^{6}$ Z.-G. Yao, X.-Q. Zhang, H.-K. Shang, X.-Y. Teng, Y.-S. Wang, and S.-H. Huang, Chin. Phys. 14, 1205 (2005).

${ }^{7}$ C. Roy, S. Byrne, E. McGlynn, J. P. Mosnier, E. dePosada, D. O’Mahony, J. G. Lunney, M. O. Henry, B. Ryan, and A. A. Cafolla, Thin Solid Films 436, 273 (2003); E. McGlynn, J. Fryar, G. Tobin, C. Roy, M. O. Henry, J.-P. Mosnier, E. de Posada, and J. G. Lunney, ibid. 458, 330 (2004).

${ }^{8}$ J. Fryar, E. McGlynn, M. O. Henry, and J. P. Mosnier, Nanotechnology 16, 2625 (2005).

${ }^{9}$ G. Tobin, E. McGlynn, M. O. Henry, J. P. Mosnier, J. G. Lunney, D. O'Mahony, and E. dePosada, Physica B 340, 245 (2003).

${ }^{10}$ A. Mitra and R. K. Thareja, J. Appl. Phys. 89, 2025 (2001).

${ }^{11}$ B. K. Meyer, H. Alves, D. M. Hofmann, W. Kriegseis, D. Forster, F. Bertram, J. Christen, A. Hoffmann, M. Strassburg, M. Dworzak, U. Haboeck, and A. V. Rodina, Phys. Status Solidi B 241, 231 (2004).

${ }^{12}$ Y. Kayanuma, Phys. Rev. B 38, 9797 (1988).

${ }^{13}$ E. McGlynn, J. Fryar, M. O. Henry, J. P. Mosnier, J. G. Lunney, D. O'Mahony, and E. de Posada, Physica B 342, 230 (2003).

${ }^{14}$ D. M. Bagnall, Y. F. Chen, M. Y. Shen, Z. Zhu, T. Goto, and T. Yao, J. Cryst. Growth 184, 605 (1998).

${ }^{15}$ P. Yu, Z. K. Tang, G. K. L. Wong, M. Kawasaki, A. Ohtomo, H. Koinuma, and Y. Segawa, J. Cryst. Growth 184, 601 (1998).

${ }^{16} \mathrm{C}$. Klingshirn, Semiconductor Optics, 2nd ed. (Springer, Berlin, 2005).

${ }^{17}$ H. Priller, J. Brückner, Th. Gruber, C. Klingshirn, H. Kalt, A. Waag, H. J. Ko, and T. Yao, Phys. Stat. Sol. B 241, 587 (2004).

${ }^{18}$ W. Y. Liang and A. D. Yoffe, Phys. Rev. Lett. 20, 59 (1968).

${ }^{19}$ R. L. Weiher and W. C. Tait, Phys. Rev. B 5, 623 (1972).

${ }^{20}$ J. M. Hvam, Phys. Status Solidi B 63, 511 (1974).

${ }^{21}$ D. F. Blossey, Phys. Rev. B 3, 1382 (1971).

${ }^{22}$ M. Gallant and A. ZemelKhan, Appl. Phys. Lett. 52, 1686 (1988).

${ }^{23}$ P. Yang, H. Yan, S. Mao, R. Russo, J. Johnson, R. Saykally, N. Morris, J. Pham, R. He, and H.-J. Choi, Adv. Funct. Mater. 12, 323 (2002). 